Pacific Journal of Mathematic 


\title{
THE STRONG APPROXIMATION THEOREM AND LOCALLY BOUNDED TOPOLOGIES ON $F(X)$
}

\author{
Jo-ANN CoHen
}

To within equivalence, the only valuations on the field $F(X)$ of rational functions over $F$ that are improper on $F$ are the valuations $v_{p}$, where $p$ is a prime polynomial of $F[X]$, and the valuation $v_{\infty}$, defined by the prime polynomial $X^{-1}$ of $F\left[X^{-1}\right]$. It is classic that if $F$ is a finite field, the set $\mathscr{P}^{\prime}$ defined by, $\mathscr{P}^{\prime}=\{p: p$ is a prime polynomial over $F\} \cup\{\infty\}$, has the Strong Approximation Property, that is, for any finite subset $G$ of $\mathscr{P}$, any $q \in \mathscr{P} \backslash G$, any family $\left(a_{g}\right)_{g \in G}$ of elements of $F(X)$ indexed by $G$, and any $M>0$, there exists a nonzero element $h$ in $F(X)$ such that $v_{p}\left(h-a_{p}\right)>M$ for all $p$ in $G$ and $v_{p}(h) \geqq 0$ for all $p$ in $\mathscr{P} \backslash(G \cup\{q\})$. We shall first prove that $\mathscr{P} \prime$ satisfies this condition when $F$ is infinite as well. We then apply this result to obtain a characterization of all locally bounded topologies on $F(X)$ for which the subfield $F$ is bounded.

1. The strong approximation theorem. Here, $\mathscr{P}$ is the set of prime polynomials in $F[X]$ and $\mathscr{P}^{\prime}$ is the set $\mathscr{P} \cup\{\infty\}$.

THEOREM 1 (The strong approximation theorem). For any finite subset $G$ of $\mathscr{P}^{\prime}$, any $q \in \mathscr{P}^{\prime} \backslash G$, any family $\left(a_{g}\right)_{g \in G}$ of elements of $F(X)$ indexed by $G$, and any positive number $M$, there exists a nonzero $h$ in $F(X)$ such that $v_{p}\left(h-a_{p}\right) \geqq M$ for all $p \in G$ and $v_{d}(h) \geqq 0$ for all $d \in \mathscr{P}^{\prime} \backslash(G \cup\{q\})$.

Proof. Let $S=\mathscr{P}^{\prime} \backslash\{q\}$. By [5, Theorem 2.2, p. 322], it suffices to show that for distinct elements $r$ and $s$ in $S$ and $M>0$, there exists an $h$ in $F(X)$ such that $v_{r}(h-1)>M, v_{s}(h)>M$ and $v_{d}(h) \geqq 0$ for all $d \in S \backslash\{r, s\}$.

Case 1. $\infty \notin S$. Then $r$ and $s$ are distinct prime polynomials and so there exist polynomials $f$ and $g$ in $F[X]$ such that $f r^{M+1}+$ $g s^{M+1}=1$. Define $h$ by, $h=g s^{M+1}$. Then $h-1=-f r^{M+1}$ and so $v_{r}(h-1) \geqq M+1>M$. Furthermore, $v_{s}(h) \geqq M+1>M$. As $h$ is a polynomial in $F[X], v_{d}(h) \geqq 0$ for all $d \in \mathscr{P}^{\circ}$ and so in particular $v_{d}(h) \geqq 0$ for all $d \in S \backslash\{r, s\}$.

Case 2. $r=\infty$. Then $s$ and $q$ are distinct prime polynomials in $F[X]$. As $v_{\infty}$ and $v_{s}$ are independent valuations on $F(X)$, there exist polynomials $f$ and $g$ such that $v_{\infty}(f / g-1)>M$ and $v_{s}(f / g)>M$ 
[1, Theorem 1, p. 134]. Choose a positive integer $t$ such that $t \operatorname{deg} q>(M+1) \operatorname{deg} s+\operatorname{deg} f+M$. By the division algorithm, there exist polynomials $w$ and $z$ in $F[X]$ such that $q^{t}=w s^{M+1} g+z$ where $\operatorname{deg} z<(M+1) \operatorname{deg} s+\operatorname{deg} g$. So $f q^{t}=f w s^{M+1} g+f z$ and hence $f / g=f w s^{M+1} / q^{t}+f z / q^{t} g$. Let $h$ be defined by $h=f w s^{M+1} / q^{t}$. Then $v_{s}(h) \geqq M+1>M$ and for all prime polynomials $p$ which are distinct from $q, v_{p}(h) \geqq 0$. So it suffices to show that $v_{\infty}(h-1)>M$.

Observe that $v_{\infty}(f / g-h)=v_{\infty}\left(f z / g q^{t}\right)=\operatorname{deg} g+t \operatorname{deg} q-\operatorname{deg} f-$ $\operatorname{deg} z>\operatorname{deg} g+(M+1) \operatorname{deg} s+\operatorname{deg} f+M-\operatorname{deg} f-(M+1) \operatorname{deg} s-$ $\operatorname{deg} g=M$. Therefore $v_{\infty}(h-1)=v_{\infty}(h-f / g+f / g-1) \geqq \min \left\{v_{\infty}(h-f / g)\right.$, $\left.v_{\infty}(f / g-1)\right\}>M$.

Case 3. $s=\infty$. Then $r$ and $q$ are distinct prime polynomials. Let $f$ be a polynomial such that $v_{r}(f-1)>M$. Choose a positive integer $t$ such that $t \operatorname{deg} q>(M+1) \operatorname{deg} r+M$. By the division algorithm, there exist polynomials $w$ and $z$ in $F[X]$ such that $q^{t} f=w r^{M+1}+z$ where $\operatorname{deg} z<(M+1) \operatorname{deg} r$. Then $f=w r^{M+1} / q^{t}+$ $z / q^{t}$. Let $h$ be defined by $h=z / q^{t}$. Then $v_{r}(f-h)=v_{r}\left(w r^{M+1} / q^{t}\right) \geqq$ $M+1>M$ and so $v_{r}(h-1)=v_{r}(h-f+f-1) \geqq \min \left\{v_{r}(h-f)\right.$, $\left.v_{r}(f-1)\right\}>M$. Furthermore,

$$
v_{\infty}(h)=t \operatorname{deg} q-\operatorname{deg} z>(M+1) \operatorname{deg} r+M-(M+1) \operatorname{deg} r=M .
$$

Finally for $d \in \mathscr{P} \backslash\{q\}, v_{d}(h) \geqq 0$.

Case 4. $\infty \in S \backslash\{r, s\}$. Then $r, s$ and $q$ are distinct prime polynomials in $F[X]$. By Case 1 , there exists a polynomial $f$ in $F[X]$ such that $v_{r}(f-1)>M$ and $v_{s}(f)>M$. Choose $t$ so large such that $t \operatorname{deg} q>(M+1)(\operatorname{deg} r+\operatorname{deg} s)$ and let $w$ and $z$ be polynomials in $F[X]$ such that $f q^{t}=w r^{M+1} s^{M+1}+z$ where $\operatorname{deg} z<(M+1)(\operatorname{deg} r+$ $\operatorname{deg} s)$. Then $f=w r^{M+1} s^{M+1} / q^{t}+z / q^{t}$. Define $h$ by $h=z / q^{t}$. Then $v_{r}(f-h)=v_{r}\left(w r^{M+1} s^{M+1} / q^{t}\right) \geqq M+1>M$ and similarly $v_{s}(f-h)>M$. Hence $v_{r}(h-1)>M$ and $v_{s}(h)>M$. Furthermore for all polynomials $p$ in $\mathscr{P} \backslash\{q\}, v_{p}(h) \geqq 0$. So it suffices to show that $v_{\infty}(h) \geqq 0$. As $v_{\infty}(h)=t \operatorname{deg} q-\operatorname{deg} z>(M+1)(\operatorname{deg} r+\operatorname{deg} s)-(M+1)(\operatorname{deg} r+\operatorname{deg} s)=0$, $v_{\infty}(h) \geqq 0$.

2. Locally bounded topologies on $F(X)$. Let $R$ be a ring and let $\mathscr{T}$ be a ring topology on $R$ (that is, $\mathscr{T}$ is a topology on $R$ making $(x, y) \rightarrow x-y$ and $(x, y) \rightarrow x y$ continuous from $R \times R$ to $R$ ). A subset $S$ of $R$ is bounded for $\mathscr{T}$ if given any neighborhood $V$ of 0 , there exists a neighborhood $U$ of 0 such that $S U \subseteq V$ and $U S \subseteq V$. S $\mathscr{T}$ is a locally bounded topology on $R$ if there is a bounded neighborhood of 0 for $\mathscr{T}$. 
A norm $\|\cdot\|$ on a field $K$ is a function from $K$ to the nonnegative reals satisfying $\|x\|=0$ if and only if $x=0,\|x-y\| \leqq\|x\|+$ $\|y\|$ and $\|x y\| \leqq\|x\|\|y\|$ for all $x, y$ in $K$. Observe that a subset of $K$ is bounded in norm if and only if it is bounded for the topology defined by the norm; in particular the topology defined by a norm is a locally bounded topology.

A subset $I$ of a field $K$ is an almost order of $K$ if (1) $0,1 \in I$, (2) $-I \subseteq I$, (3) $I I \subseteq I$, (4) there exists a nonzero element $h$ in $I$ such that $h(I+I) \subseteq I$, and (5) for each $x \in K^{*}$, there exist $y, z$ in $I^{*}$ such that $x=y z^{-1}$.

Lemma 1 [6, Theorems 5 and 6]. If $\mathscr{T}$ is a nondiscrete, locally bounded ring topology on a field $K$, then there is an almost order $I$ of $K$ that is a bounded neighborhood of zero. Conversely, if $I$ is an almost order of $K$, then there exists a unique nondiscrete, locally bounded ring topology $\mathscr{T}$ on $K$ for which $I$ is a bounded neighborhood of zero. Furthermore, the topology $\mathscr{T}$ defined by $I$ is Hausdorff if and only if $I \neq K$.

In [7] we investigated locally bounded topologies on the quotient fields of certain Dedekind domains. We recall the results of that paper.

Let $K$ be the quotient field of a Dedekind domain $R$ that is not a field, $\mathscr{P}$ the set of nonzero prime ideals of $R$ and $\mathscr{P}_{\infty}$ a set $\left\{|\cdot|_{1}, \cdots,|\cdot|_{n}\right\}$ of $n$ mutually inequivalent proper absolute values on $K$ such that for each $i \in[1, n]$ and each $p \in \mathscr{P}$, the topology $\mathscr{T}_{i}$ defined by $|\cdot|_{i}$ is distinct from the topology $\mathscr{T}_{p}$ defined by the valuation $v_{p}$. Let $\mathscr{P}^{\prime}$ be defined by $\mathscr{P}^{\prime}=\mathscr{P}^{\prime} \cup \mathscr{P}_{\infty}$. For each subset $S$ of $\mathscr{P}^{\prime}$, we define $O(S)$ by $O(S)=\left\{x \in K: v_{p}(x) \geqq 0\right.$ for all $p \in S \cap \mathscr{P}$ and $|x|_{i} \leqq 1$ for all $\left.|\cdot|_{i} \in S \cap \mathscr{P}_{\infty}\right\}$.

We placed the following conditions on $K, R$ and $\mathscr{P}^{\prime}$ :

I. Class Number Condition (CC). The class number of $K$ over $R$ is finite.

II. Approximation Condition (AC). For any finite subset $G$ of $\mathscr{P}^{\prime}$, any $\gamma \in \mathscr{P}^{\prime} \backslash G$, any family $\left(a_{g}\right)_{g \in G}$ of elements of $K$ indexed by $G$, and any positive numbers $M$ and $e$, there exists a nonzero element $h$ in $K$ such that $v_{p}\left(h-a_{p}\right) \geqq M$ for all $p \in G \cap \mathscr{P}, \mid h-a_{\left.|\cdot|_{k}\right|_{2}} \leqq e$ for all $|\cdot|_{2} \in G \cap \mathscr{P}_{\infty}$ and $h \in O\left(\mathscr{P}^{\prime} \backslash(G \cup\{\gamma\})\right)$.

III. Discreteness Condition (DC). The only ring topology on $K$ for which $O\left(\mathscr{P}^{\prime}\right)$ is a neighborhood of zero is the discrete topology. 
IV. Euclidean Condition (EC). There exist positive numbers $\beta_{1}, \cdots, \beta_{n}$ such that if $a, b \in R$ with $b \neq 0$, then there exist $q, r$ in $R$ satisfying $a=b q+r,|r|_{i} \leqq|b|_{i} \beta_{i}$ for all $i$ in $[1, n]$.

Lemma 2 [7, Lemma 2]. If $S$ is a nonempty, proper subset of $\mathscr{P}^{\prime}$, then $O(S)$ is an almost order for a unique, Hausdorff, nondiscrete, locally bounded ring topology $\mathscr{T}_{s}$ on $K$.

Lemma 3 [7, Theorem 3, Statement 3]. Let $\mathscr{T}$ be a Hausdorff, nondiscrete, locally bounded ring topology on $K$ with the following property.

V. Boundedness Condition (BC). For any $M>0$, the set $O(\mathscr{P}) \cap\left\{y \in K:|y|_{i} \leqq M\right.$ for all $\left.|\cdot|_{i} \in \mathscr{P}_{\infty}\right\}$ is a bounded set for $\mathscr{T}$.

If $\mathscr{P}_{\infty}$ has exactly one element, then $\mathscr{T}^{-}=\mathscr{T}_{S}$ for some nonempty, proper subset $S$ of $\mathscr{P}^{\prime}$.

THEOREm 2. Let $F$ be a field and $X$ an indeterminate over $F$. Let $\mathscr{P}$ be the set of all prime polynomials over $F, v_{\infty}$ the valuation on $F(X)$ defined by $v_{\infty}(f / g)=\operatorname{deg} g-\operatorname{deg} f$ and let $\mathscr{P}_{\infty}=\left\{|\cdot|_{\infty}\right\}$ where $|y|_{\infty}=2^{-v_{\infty}(y)}$ for all $y$ in $F(X)$. Then $F(X), F[X]$ and $\mathscr{P}^{\prime}=\mathscr{P} \cup \mathscr{P}_{\infty}$ satisfy (CC), (AC), (DC) and (EC). Moreover, if $\mathscr{T}$ is a Hausdorff, nondiscrete, locally bounded ring topology on $F(X)$ for which the subfield $F$ is bounded, then $\mathscr{T}$ satisfies (BC) and hence $\mathscr{T}=\mathscr{T}_{S}$ for some nonempty, proper subset $S$ of $\mathscr{P}^{\prime}$.

Proof. As $F[X]$ is a principal ideal domain, (CC) holds. By Theorem 1, (AC) holds. Furthermore, (DC) holds. Indeed, as $O\left(\mathscr{P}^{\prime}\right)=F$, if $\mathscr{T}$ is a ring topology on $F(X)$ for which $O\left(\mathscr{P}^{\prime}\right)$ is a neighborhood of zero, then the set $F \cap F X=\{0\}$ is a neighborhood of zero for $\mathscr{T}$. Thus $\mathscr{T}$ is discrete. By the division algorithm, (EC) holds with $\beta_{1}=1$. So it suffices to prove that (BC) holds when $\mathscr{T}$ is a locally bounded topology on $F(X)$ for which the subfield $F$ is bounded.

Notice that for $M>0, O(\mathscr{P}) \cap\left\{y \in F(X):|y|_{\infty} \leqq M\right\}=\{y \in F[X]:$ $\operatorname{deg} y \leqq N\}$ where $N=\ln M / \ln 2$. Consequently, if $\mathscr{T}$ is a locally bounded topology on $F(X)$ for which the subfield $F$ is bounded, then $\mathscr{T}$ satisfies (BC) and therefore by Lemma 3, $\mathscr{T}=\mathscr{T}_{S}$ for some nonempty, proper subset $S$ of $\mathscr{P}^{\prime}$.

Corollary [7, Corollary 4]. If $F$ is a finite field and $\mathscr{T}$ is a Hausdorff, nondiscrete, locally bounded topology on $F(X)$, then there exists a nonempty, proper subset $S$ of $\mathscr{P}^{\prime}$ such that $\mathscr{T}=\mathscr{T}_{S}$. 
The following theorem is a generalization of Theorem 2 of [3].

THEOREM 3. Let $\mathscr{T}$ be a Hausdorff, nondiscrete, locally bounded ring topology on $F(X)$ for which the subfield $F$ is bounded. The following statements are equivalent.

$1^{\circ} \mathscr{T}$ is a field topology.

$2^{\circ} \mathscr{T}$ is the supremum of finitely many valuation topologies $\mathscr{T}_{p}$ where $p \in \mathscr{P}^{\prime}$.

$3^{\circ}$ There exists a nonzero element $a$ in $F(X)$ such that $\lim _{n \rightarrow \infty} a^{n}=0$.

$4^{\circ} \mathscr{T}$ is defined by a norm.

Proof. Let $S$ be a nonempty, proper subset of $\mathscr{P}^{\prime}$ such that $\mathscr{T}=\mathscr{T}_{s}$.

To show that $1^{\circ}$ implies $2^{\circ}$, it suffices to show that $S$ is finite. As $\mathscr{T}$ is a field topology and $O(S)+1$ is a neighborhood of 1 in $\mathscr{T}$, there exists a $y$ in $O(S) \backslash\{0\}$ such that $(y O(S)+1)^{-1} \cong O(S)+1$. If $S$ is infinite, pick $p \in S \cap \mathscr{P}$ such that $v_{p}(y)=0$. By Theorem 1 , there exists a $z$ in $F(X)$ such that $v_{p}\left(z+y^{-1}\right)>0$ and $z \in O(S \backslash\{p\})$. Then $v_{p}(z)=v_{p}\left(z+y^{-1}-y^{-1}\right) \geqq \min \left\{v_{p}\left(z+y^{-1}\right), v_{p}\left(y^{-1}\right)\right\} \geqq 0$ and so $z \in O(S)$. Hence $y z+1 \in y O(S)+1$ and $v_{p}(y z+1)=v_{p}\left(y\left(z+y^{-1}\right)\right)=$ $v_{p}(y)+v_{p}\left(y+z^{-1}\right)>0$. Therefore, $v_{p}\left((y z+1)^{-1}\right)<0$. But $(y z+1)^{-1} \in$ $O(S)+1$ and $v_{p}(w) \geqq 0$ for all $w \in O(S)+1$. Contradiction! Therefore $S$ is finite.

To prove that $2^{\circ}$ implies $3^{\circ}$, we note that if $S$ is any nonempty, finite subset of $\mathscr{P}^{\prime}$ and $a$ is any nonzero element of $F(X)$ such that $|a|_{\infty}<1$ when $|\cdot|_{\infty} \in S$ and $v_{p}(a)>0$ for all $p$ in $S \cap \mathscr{P}$, then $\lim _{n \rightarrow \infty} a^{n}=0$ in $\mathscr{T}_{s}$. The existence of such an element is guaranteed by Theorem 1 . The statement $3^{\circ}$ implies $4^{\circ}$ is a special case of Cohn's theorem [4, Theorem 6.1]. Finally the proof that $4^{\circ}$ implies $1^{\circ}$ is the same as that for normed algebras found on p. 77 of [2].

\section{REFERENCES}

1. N. Bourbaki, Algèbre Commutative, Ch. 5-6, Hermann, Paris, 1964.

2. - Topologie Générale, Ch. 9, Paris, Hermann, 1958.

3. J. Cohen, Locally bounded topologies on $F(X)$, Pacific J. Math., 70 (1977), 125-132.

4. P. M. Cohn, An invariant characterization of pseudo-valuations on a field, Proc. Cambridge Phil. Soc., 50 (1954), 159-177.

5. J. Goldhaber and G. Ehrlich, Algebra, The MacMillan Company, London, 1970.

6. Hans-Joachim Kowalsky and Hansjürgen Dürbaum, Arithmetische Kennzeichung von Korpertopologien, J. Reine Angew. Math., (1953), 135-152.

7. E. Nichols and J. Cohen, Locally bounded topologies on global fields, Duke Math. J., 44 (1977), 853-862.

8. O. T. O'Meara, Introduction to Quadratic Forms, Springer-Verlag, Berlin, 1963.

Received May 15, 1978.

North Carolina State University

RALEIGH, NC 27650 



\section{PACIFIC JOURNAL OF MATHEMATICS}

\section{EDITORS}

DONALD BABBITT (Managing Editor)

University of Galifornia

Los Angeles, California 90024

HUGo RossI

University of Utah

Salt Lake City, UT 84112

C. C. MOORE AND ANDREW OGG

University of California

Berkeley, CA 94720

\section{J. DUGUNDJI}

Department of Mathematics University of Southern California Los Angeles, California 90007

R. FINN AND J. MILGRAM Stanford University Stanford, California 94305

ASSOCIATE EDITORS
E. F. BECKENBACH
B. H. NeumanN
F. WoLF
K. YosHIDA

\section{SUPPORTING INSTITUTIONS}

UNIVERSITY OF BRITISH COLUMBIA UNIVERSITY OF SOUTHERN CALIFONIA CALIFORNIA INSTITUTE OF TECHNOLOGY UNIVERSITY OF CALIFORNIA MONTANA STATE UNIVERSITY STANFORD UNIVERSITY UNIVERSITY OF HAWAII UNIVERSITY OF TOKYO UNIVERSITY OF NEVADA, RENO UNIVERSITY OF UTAH NEW MEXICO STATE UNIVERSITY WASHINGTON STATE UNIVERSITY OREGON STATE UNIVERSITY UNIVERSITY OF OREGON UNIVERSITY OF WASHINGTON 


\section{Pacific Journal of Mathematics \\ Vol. 87, No. $1 \quad$ January, 1980}

Spiros Argyros, A decomposition of complete Boolean algebras ..........

Gerald A. Beer, The approximation of upper semicontinuous multifunctions

by step multifunctions . . . . ....................

Ehrhard Behrends and Richard Evans, Multiplicity theory for Boolean

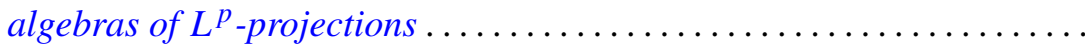

Man-Duen Choi, The full $C^{*}$-algebra of the free group on two

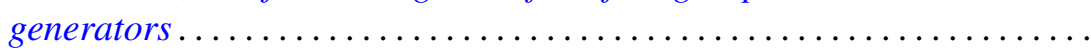

Jen-Chung Chuan, Axioms for closed left ideals in a $C^{*}$-algebra . . . . . . . .

Jo-Ann Deborah Cohen, The strong approximation theorem and locally

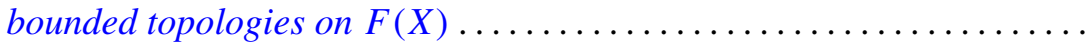

Eugene Harrison Gover and Mark Bernard Ramras, Increasing sequences of Betti numbers............................

Morton Edward Harris, Finite groups having an involution centralizer with

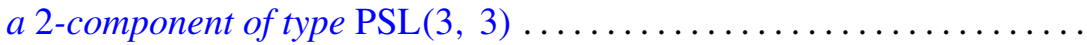

Valéria Botelho de Magalhães Iório, Hopf $C^{*}$-algebras and locally compact

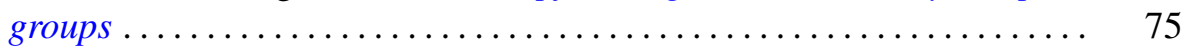

Roy Andrew Johnson, Nearly Borel sets and product measures . . . . . . . . . .

Lowell Edwin Jones, Construction of $Z_{p}$-actions on manifolds . . . . . . . . .

Manuel Lerman and Robert Irving Soare, $d$-simple sets, small sets, and

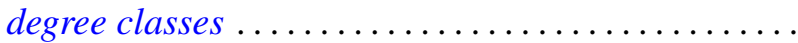

Philip W. McCartney, Neighborly bushes and the Radon-Nikodým property

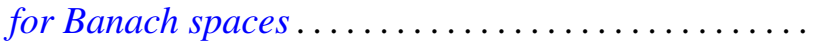

Robert Colman McOwen, Fredholm theory of partial differential equations on complete Riemannian manifolds.

Ernest A. Michael and Carl Preston Pixley, A unified theorem on continuous selections.

Ernest A. Michael, Continuous selections and finite-dimensional sets .

Vassili Nestoridis, Inner functions: noninvariant connected components...

Bun Wong, A maximum principle on Clifford torus and nonexistence of proper holomorphic map from the ball to polydisc.

Steve Wright, Similarity orbits of approximately finite $C^{*}$-algebras . . .

Kenjiro Yanagi, On some fixed point theorems for multivalued

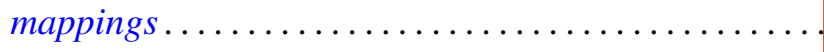

Wieslaw Zelazko, A characterization of LC-nonremovable ideals in commutative Banach algebras 